\title{
Dynamic gut microbiome changes following regional intestinal lymphatic obstruction in primates
}

\author{
F. Becker ${ }^{\mathrm{a}, \mathrm{b}, 1}$, F.N.E. Gavins ${ }^{\mathrm{a}, 1}$, J. Fontenot ${ }^{\mathrm{c}}$, P. Jordan ${ }^{\mathrm{d}}$, J.Y. Yun ${ }^{\mathrm{a}}$, R. Scott ${ }^{\mathrm{e}}$, P.R. Polk ${ }^{\mathrm{f}}$, \\ R.E. Friday ${ }^{g}$, M. Boktor ${ }^{d}$, M. Musso ${ }^{c}$, E. Romero ${ }^{c}$, S. Boudreaux ${ }^{c}$, J. Simmons ${ }^{\text {h }}$, \\ D.L. Hasselschwert ${ }^{c}$, J.E. Goetzmann ${ }^{c}$, J. Vanchiere ${ }^{i}$, U. Cvek $^{j}$, M. Trutschl $^{\mathrm{j}}$, P. Kilgore $^{\mathrm{j}}$, \\ J.S. Alexander ${ }^{\mathrm{a}, e, *}$ \\ a Department of Molecular and Cellular Physiology, Louisiana State University Health Sciences Center Shreveport, Shreveport, LA, USA \\ ${ }^{\mathrm{b}}$ Department for General-Visceral and Transplant Surgery, University of Münster, Münster, Germany \\ c University of Louisiana at Lafayette, New Iberia Research Center, New Iberia, LA, USA \\ d Louisiana State University School of Medicine, Department of Gastroenterology and Hepatology, Shreveport, LA, USA \\ e Department of Microbiology and Immunology, Louisiana State University Health Sciences Center Shreveport, Shreveport, LA, USA \\ ${ }^{\mathrm{f}}$ Research Core Facility, Louisiana State University Health Sciences Center Shreveport, Shreveport, LA, USA \\ ${ }^{g}$ Feist-Weiller Cancer Center, Louisiana State University Health Sciences Center Shreveport, Shreveport, LA, USA \\ ${ }^{\mathrm{h}}$ Keeling Center for Comparative Medicine and Research, The University of Texas MD Anderson Cancer Center, Texas, TX, USA \\ ${ }^{i}$ Department of Pediatrics, Section of Pediatric Infectious Diseases, Louisiana State University Health Sciences Center - Shreveport, Shreveport, LA, USA \\ j Department of Computer Sciences, Louisiana State University-Shreveport, Shreveport, LA, USA
}

\section{A R T I C L E I N F O}

\section{Article history:}

Received 18 February 2019

Received in revised form 24 May 2019

Accepted 26 June 2019

Available online $\mathrm{xxx}$

\section{Keywords:}

Inflammatory bowel disease

Crohn's disease

Non-human primate

Lymphatics

Microbiome

Lymphatic obstruction

\begin{abstract}
A B S T R A C T
The pathogenesis of inflammatory bowel disease (IBD) has been linked with lymphostasis, but whether and how lymphatic obstruction might disturb the intestinal microbiome in the setting of Crohn's Disease (CD) is currently unknown. We employed a new model of CD in African Green monkeys, termed 'ATLAS' ( African green monkey truncation of lymphatics with obstruction and sclerosis), to evaluate how gut lymphatic obstruction alters the intestinal microbiome at 7,21 and 61 days. Remarkable changes in several microbial sub- groupings within the gut microbiome were observed at 7 days post-ATLAS compared to controls including increased abundance of Prevotellaceae and Bacteroidetes-Prevotella-Porphyromonas (BPP), which may contribute to disease activity in this model of gut injury. To the best of our knowledge, these findings represent the first report linking lymphatic structural/gut functional changes with alterations in the gut microbiome as they may relate to the pathophysiology of $C D$.
\end{abstract}

(c) 2019 Elsevier B.V. All rights reserved.

\section{Introduction}

Alterations in the gut microbiota composition (dysbiosis) are well-recognized contributors to the pathogenesis of gastrointestinal disorders, such as inflammatory bowel diseases (IBD), including ulcerative colitis (UC) and Crohn's disease (CD). In addition, a growing body of evidence from clinical data as well as several animal models have been implicated the contribution of lymphatic obstruction towards CD pathogenesis [1]. However, significant dif-

\footnotetext{
* Corresponding author at: Louisiana State University Health Sciences Center Shreveport Department of Molecular and Cellular Physiology, 1501 Kings Highway, Shreveport, LA 71130-3932, USA.

E-mail address: jalexa@lsuhsc.edu (J.S. Alexander).

1 These authors contributed equally.
}

ferences in gut anatomy between humans and other species [2] differences in diets [3] and inherent differences in gut flora between species [3-5] have confounded interpretations of how lymphatics may govern gut homeostasis. These studies have shown that lymphatic blockage intensifies inflammation, but it is still unclear how such induced inflammation might in turn modulate the microbiome.

Few, if any IBD models fully recapitulate clinical features found in $\mathrm{CD}$, and experimental models which more faithfully replicate this condition are still needed. The recent availability of African Green monkey (Chlorcebus aethiops) models for this collaboration provided a unique opportunity to study one of the most humanlike examples of intestinal structure in a model of $\mathrm{CD}$. This new model, termed 'African green monkey truncation of lymphatics with obstruction and sclerosis' ('ATLAS'), allowed us to evaluate for the first time how surgically-induced regional intestinal lym- 
phatic obstruction would disturb gut structure and function and reorganization of the microbiome in a non-human primate from baseline measured at three time points over 61 days after generation of this model. We hypothesized that in addition to provoking histopathological changes, lymphatic obstruction in the ATLAS might modulate the gut microenvironment and alter the microbiome which is highly responsive to this environment.

It is well known that intestinal dysbiosis can lead to immune system activation, triggering and intensifying gut inflammation [6,7]. Such dysbiosis can arise from several causes including infection, antibiotic use, diet and altered gut motility. We have previously shown that gut sterilization during induction of dextran sodium sulfate (DSS, an irritant which produces gut epithelial injury) colitis significantly suppressed the development of tissue injury and clinical symptomatology [8], characterized by preservation of tissue architecture and suppression of intestinal blood and lymphatic network remodeling. Using this model of experimental colitis in rodents, Munyaka et al. [9] found that DSS-induced gut injury induces microbial dysbiosis.

Immune activation caused by dysbiosis has also been shown to intensify extra-intestinal forms of tissue injury including neuronal [10] and renal injury [11]. While departures from the normal composition of the gut microbiome have been repeatedly demonstrated in experimental models of and in patients with IBD, findings regarding whether and how gut injury mediates effects in the microbiome are still lacking. Non-chemically induced and more clinically relevant experimental models of IBD are necessary to illuminate how change(s) in the gut environment itself might lead to alterations of the intestinal microbiome, and how long these disturbances persist relative to the physical and functional state of the intestine. Large shifts in the microbiome which result from gut injury may represent an important pathophysiologic event which creates a vicious cycle of immune system activation leading to disease intensification in IBD.

Changes in diet provided to the members of the gut microbiome can dramatically skew the makeup of the microbiome with important consequences on signaling at the gut-microbiome interface that influence both the structural and immunological integrity of the intestinal barrier $[12,13]$. For example, the diminished availability of complex carbohydrates that can be digested by Lactobacillus and Bifidobacteria to produce short chain fatty acids (an efficient carbon source for intestinal epithelial cells (IECs)) may derange intestinal barrier function [12] and diminish the capacity of IEC to maintain mucosal tolerance via antigen presenting cell trafficking [13].

In this study, we sought to determine the duration and mechanism by which acute gut inflammation in otherwise healthy primates might provoke intestinal dysbiosis. Additionally, as these microbiome changes originated solely as a response to lymphatic obstruction, this model suggests a primary role for lymphatic flow in maintenance of intestinal homeostasis and that lymphatic obstruction may represent an important, but often overlooked component of the pathogenesis of IBD.

\section{Materials and methods}

\subsection{Animals}

All animal protocols were approved by the University of Louisiana (UL) at Lafayette Animal Care and Use Committee and were performed in accordance with the Animal Welfare Act and the National Research Council's "Guide for the Care and Use of Laboratory Animals". Male African Green monkeys (Chlorocebus aethiops sabaeus) were used and maintained at the New Iberia Research Center (NIRC), UL. Animals were fed a regular primate diet (Purina Lab,
St. Louis, MO, USA), which was supplemented with fruit 2-3 times weekly. Tap water was provided ad libitum via automatic watering device. Preoperatively, animals were fasted overnight with free access to water. Postoperatively, animals had free access to food and water ad libitum.

\subsection{The ATLAS model}

An in-depth description of the ATLAS model, along with the physiological and inflammatory data used to define the model was reported in a previous paper [14]. Briefly, animals underwent a laparotomy and intestinal and mesenteric lymphatic vessels of the distal ileum and ascending colon were identified by subserosal injections of Isosulfan blue ( $300 \mu \mathrm{l}, 1 \%$, Lymphazurin ${ }^{\mathrm{TM}}$, Covidien, New Haven, CT, USA). Next the mesenteric lymphatic vessels were ligated and the respective draining lymph nodes were sclerosed by injecting $4 \%$ formalin. Surgically induced lymphatic obstruction was deemed successful if stasis of Isosulfan blue occurred in the mesenteric lymphatics and a complete reuptake occurred in the proximal lymph nodes without any leakage. It is important to state that the ATLAS model had no mortality and that animals in the ATLAS model displayed no signs of morbidity [14].

\subsubsection{Experimental groups}

To evaluate the course of intestinal injury in acute, mid-term and chronic phases of gut injury following lymphatic obstruction and sclerosis, four different groups were analyzed: 1) control group consisting of naïve animals without any treatment $(n=20), 2)$ acute ileitis ( 7 days. $n=4), 3$ ) midterm ileitis (21 days. $n=4$ ) and 4 ) chronic ileitis (61 days. $n=4$ ). Time points were selected based on previous studies by Kalima [15]. We anticipated a direct influence of the intestinal lymphatic obstruction on the ileal clinical phenotype which could include weight loss, growth retardation, diarrhea, steatorrhea or fever. Other important key features of this model historically include fistulae, stenoses, internal obstructions or perforations, especially when the model is complicated by infections.

\subsection{Stool sample processing}

We collected feces samples from the naïve control group as well as from all experimental animals during routine examinations at the respective timepoints (7-, 21- and 61-days). We used 20 animals in the control group and collected feces from 12 animals after 7 days as well as from four animals after 21 and 61 days, respectively. Microbial DNA from stool samples was isolated using QiaAmp DNA Stool kit (Qiagen, Gaithersburg, MD) using the standard Qiacu protocol. Briefly, stool samples (average + SD weight $=0.173 \pm 0.08 \mathrm{~g}$ ) were combined with $1 \mathrm{ml}$ inhibitor in tEX buffer and vortexed for $30 \mathrm{~s}$. Samples were then heated to $950 \mathrm{C}$ for $5 \mathrm{~min}$, cooled on ice and centrifuged to pellet non-suspended fecal material. $200 \mu \mathrm{l}$ of supernatant were transferred to a new tube were and applied to QiaCube. Purified samples were eluted in a final volume of $200 \mu \mathrm{l}$ buffer ATE. DNA was quantified using a Qubit dsDNA HS assay (Life Technologies, Grand Island, NY).

\subsection{Metagenomics library preparation and sequencing}

Libraries were prepared using the Illumina 16S Metagenomics Sequencing Library Preparation protocol. Briefly, the V3 and V4 regions of the 16s rRNA gene were amplified using a limited cycle PCR with region- specific primers that included the Illumina adapter overhang sequences [16]. The primer sequences were 16S Amplicon Forward 5' TCGTCGGCAGCGTCAGATGTGTATAAGAGACAGCCTACGGGNGGCWGCAG 3' and 16S Amplicon Reverse 5' GTCTCGTGGGCTCGGAGATGTGTATAAGAGACAGGACTACHVGGGTATCTAATCC 3'. Amplifications were 
performed using $12.5 \mathrm{ng}$ of DNA template, $200 \mathrm{nM}$ primers, and KAPA HiFi Hot Start Ready Mix in a C1000 Bio-Rad thermocycler with the following cycling conditions: 1 cycle at $95^{\circ} \mathrm{C}$ for $3 \mathrm{~min}, 25$ cycles of $95^{\circ} \mathrm{C}$ for $30 \mathrm{~s}, 55^{\circ} \mathrm{C}$ for $30 \mathrm{~s}$, and $72^{\circ} \mathrm{C}$ for $30 \mathrm{~s}$, and 1 cycle at $72{ }^{\circ} \mathrm{C}$ for $5 \mathrm{~min}$. PCR reactions were performed in duplicate and then pooled prior to purification with Agencourt AMPure XP (Beckman Coulter, Inc., Brea, CA). A second PCR was performed to attach dual indices and Illumina sequencing adapters using the Nextera XT Index Kit as recommended by the manufacturer (Illumina, San Diego, CA).

Library size was determined on the Agilent TapeStation with a D1000 assay (Agilent, Santa Clara, CA). Libraries were quantified with a Qubit dsDNA HS assay and diluted to a final concentration of $4 \mathrm{pM}$. The libraries were spiked with $5 \%$ PhiX control and sequenced on an Illumina MiSeq system using a paired-end 300cycle protocol. Sequence reads passing filter ranged from 239,000 to 718,000 for each sample. Initial analyses were performed in Illumina MiSeq Reporter v2.5 using the Metagenomics workflow. This provided a taxonomic classification using the Greengenes database (http://greengenes.lbl.gov/).

\subsection{Data handling}

Several techniques were used to 'sanitize' the aggregate report. An ünclassifiedc̈ategory exists in the report that was found with a count at least two orders of magnitude greater than any classified read. This was considered as noise and removed from the data. Additionally, counts varied in magnitude across samples, so it was necessary to normalize them to account for variation in total population size. We considered four scaling methods of normalization: global, log-global, group, and species. The global method utilizes the maximum intensity, normalizes the entire data set as an upper bound and rescales the data to the interval $(0$, 1) using MinMax normalization; log-global is similar, except it uses the natural logarithm of the magnitude. While these methods can highlight areas where one species has unusually high activity, it is possible that one sample would have a very strong signal (effectively obscuring trends in the others). To account for this, we performed normalization between samples in the same group (which more accurately reveals trends occurring between them) and within the same species (showing where the peak number of reads occurred for that species). We collected the aggregate read classification data from Illumina's 16S Metagenomics v1.0.1 application to determine the distribution of microbial species in each sample. Illumina's 16S Metagenomics v1.0.1 app in BaseSpace is an extension of the metagenomics workflow found in the MiSeq Reporter Software (MSR) 2.4. The 16S Metagenomics app provides ultra-fast taxonomic classification of the bacterial 16S rRNA gene without the need for upfront OTU clustering [16]. The reads are classified against the GreenGenes database, with species-level sensitivity. The classification algorithm is based on a high-performance implementation of the Ribosomal Database Project (RDP) naïve Bayesian algorithm. The classification step uses ClassifyReads, a high-performance implementation of the RDP Classifier described in Wang et al. [17]. We derived our counts from the species report and used NCBI Taxonomy to tag each species a complete taxonomic hierarchy. This allowed us to make inferences about relationships between taxa at varying scopes.

\subsection{Microbiome analysis}

Aggregate read classification data (Illumina 16S Metagenomics v1.0.1) were applied to determine distributions of microbial taxonomic classifications of 16S rRNAs without the need for upfront OTU clustering [16]. Classification algorithms use the Ribosomal Database Project (RDP) naïve Bayesian algorithm using Classi-
fyReads, a high-performance implementation of the RDP Classifier [17]. Association testing of all covariates vs. all taxa were performed via pairwise $T$-tests of sample means supplanted by one-way ANOVA and Principal Coordinate Analysis (PCOA).

\subsubsection{S RNA sequencing to evaluate changes in the gut microbiome following ATLAS model}

We performed massive parallel sequencing of 16S RNAs using the Illumina MiSeq sequencer to identify and semi-quantitate microbial species in fecal samples from African Green monkeys. Samples were serially collected from each animal to evaluate how and which changes in the gut microbiome are induced as colitic disease develops [18]. The identification of particular species of bacteria and their relative abundance at different times in the course of disease may link particular species with features of disease and could identify microbial targets for therapy which if suppressed (or expanded) might reduce disease activity in experimental and human IBD. Counts for species reports used NCBI Taxonomy to tag each species and generate a taxonomic hierarchy. This allowed us to make inferences regarding relationships between taxa under different circumstances.

\subsection{Statistical analysis}

Association testing of all covariates versus all taxa was performed via pairwise t-tests of sample means supplanted by one-way ANOVA and Principal Coordinate Analysis (PCOA). Statistical analysis was conducted with version 7, GraphPad Software,San Diego, CA) and data were expressed as average \pm SEM with a $\mathrm{p}<0.05$ being considered significant.

\section{Results}

\subsection{Lymphatic obstruction altered species diversity of the microbiome}

The proportional contribution of each bacterial species to the composition of the total microbiome in each sample was displayed using a 'heat map' strategy (Fig. 1), where only species which were within the top $85 \%$ of total classified DNA reads were studied. The remaining $\tilde{1} 5 \%$ of the species were not included in this analysis because of the low relative contribution of each component in this fraction. Fig. 1 shows species (list shown on left) ordered by their taxonomic relationship according to NCBI with more closely related species being grouped together. The scale shows the percentage of maximum DNA reads per treatment group with a color scaling gradient separation of 5\%. Species are ordered horizontally (in rows), individually analyzed samples are represented in columns.

These data show that there was a remarkable reduction in the diversity of the gut microbiome at day 7 following intestinal lymphatic obstruction, compared to controls and compared to day 21 and day 61 post- operatively (Fig. 1B). Microbial diversity appears to be 'restored' at days 21 and 61, but this repopulation appears to represents a different set of commensal bacteria which comprising the 'new' microbiome.

\subsection{Intestinal lymphatic obstruction changes the composition of the microbiome at the phylum level}

Fig. 2 shows that the abundance of the members in the four major microbial phyla (Bacterioidetes, Firmicutes, Spirochaetes, and Proteobacteria) changed dramatically as a function of time after induction of the ATLAS model. Fig. 2A shows changes in the abundance of each phylum as a function of time following ATLAS compared to control groups. In this graph, the length of each cell in the bar graph is proportionate to the total classified DNA reads 


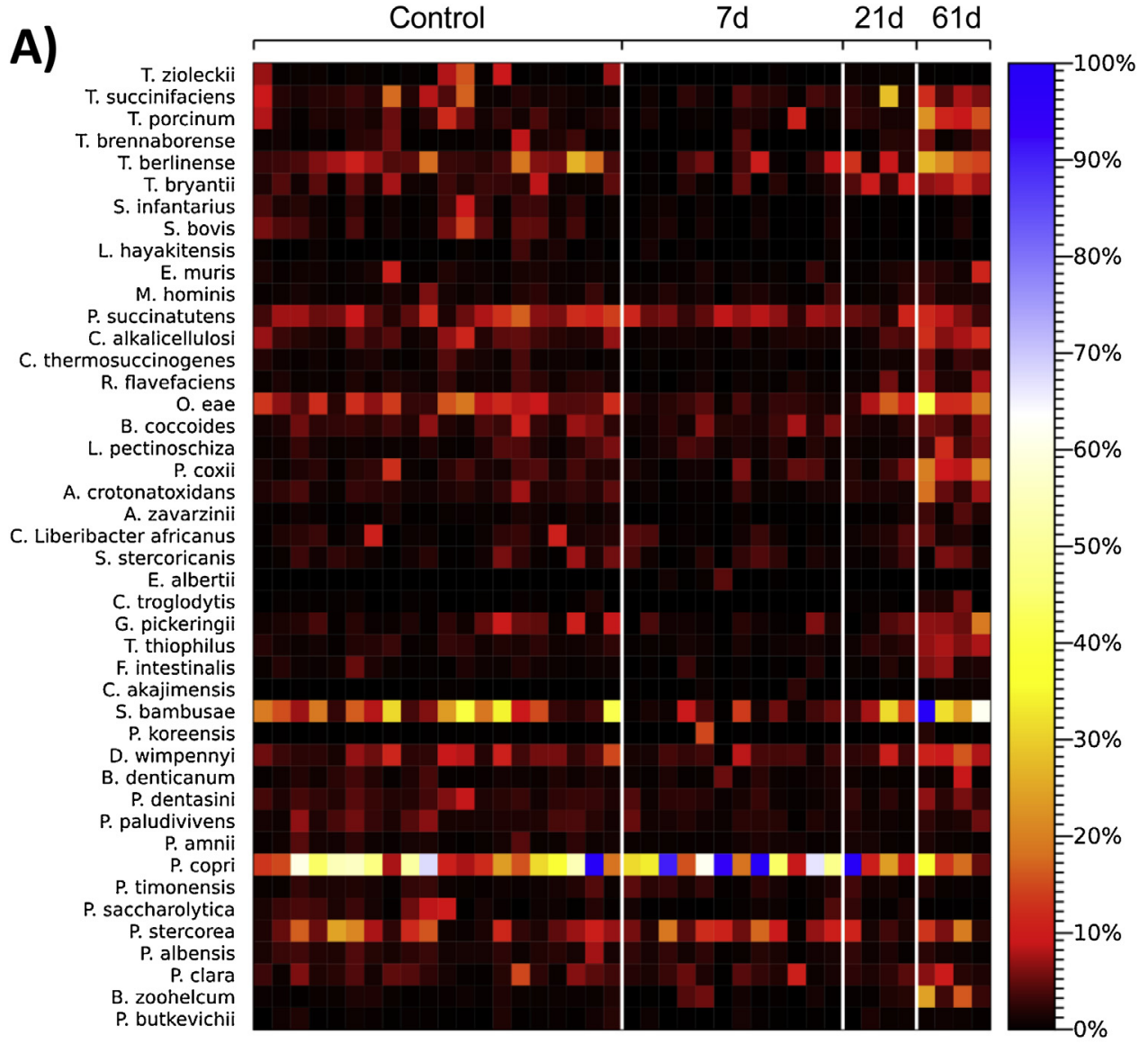

B)

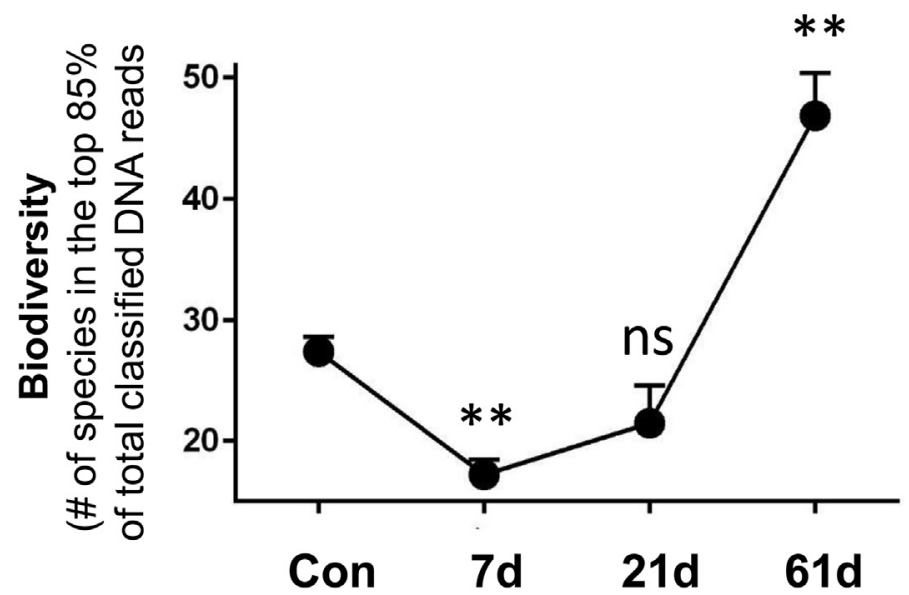

Fig. 1. Intestinal lymphatic obstruction altered species diversity of the gut bacterial microbiome.

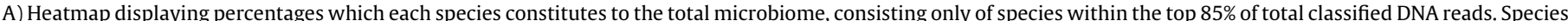

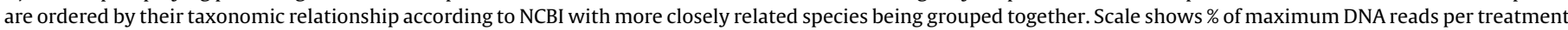

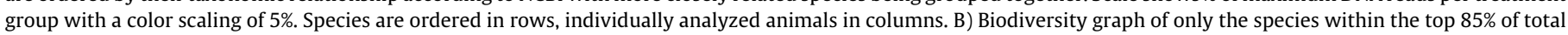
classified DNA reads for control, 7, 21 and 61 days respectively ( $\mathrm{n}=20$ for control, $n=12$ for 7 days and $n=4$ for treatment groups of 21 days and 61 days).

for that phylum within that treatment group. We found that there was an expansion in the Bacteriodetes and a reduction in the Firmicutes such that the Log2 ratio of Firmicutes to Bacteriodetes showed a decrease in this ratio at day 7 , with a restoration by day 21 and maintenance at day 61 (Fig. 2B).

\subsection{Intestinal lymphatic obstruction changed the composition of the intestinal microbiome at the family level}

Fig. 3A shows the changes in the abundance of particular families of commensal bacteria as a function of time following ATLAS. 
A)

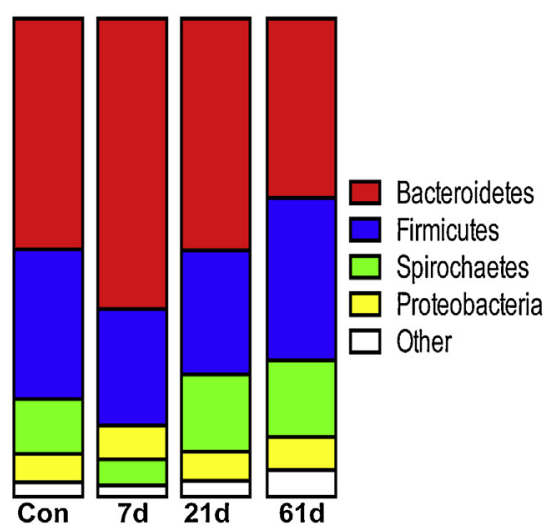

B)

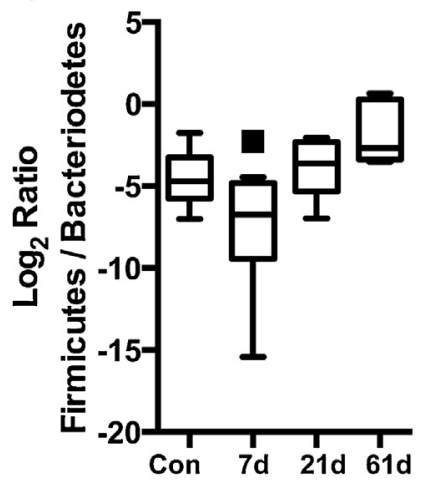

Fig. 2. Intestinal lymphatic obstruction changed the composition of the microbiome at phylum level.

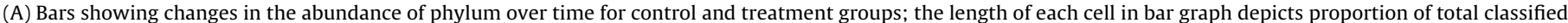

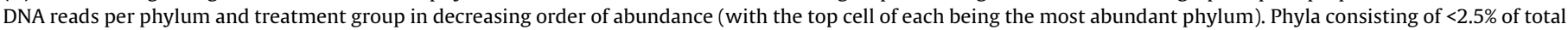

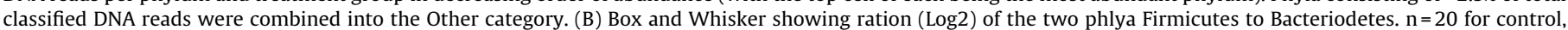

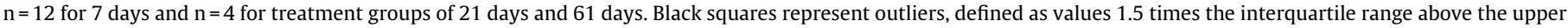
quartile.

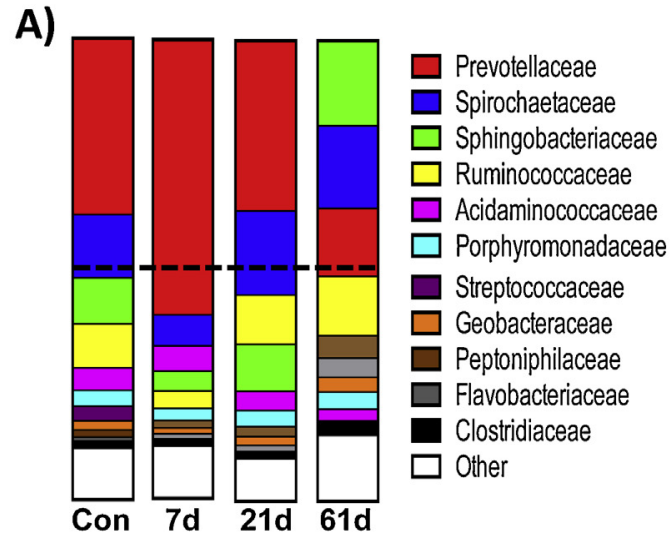

B)

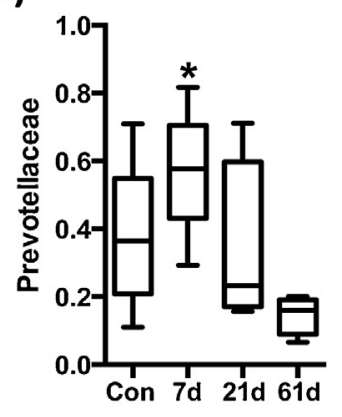

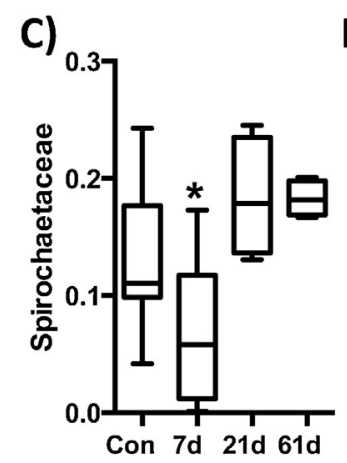

D)

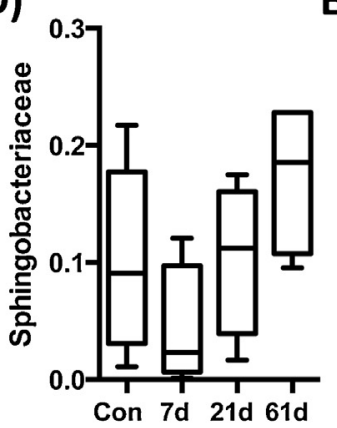

E)

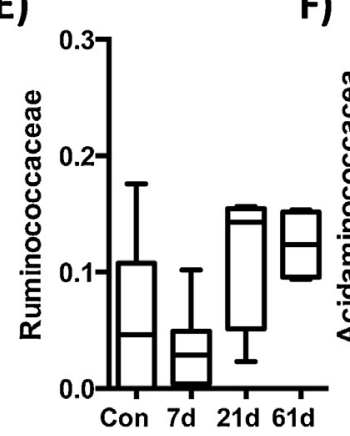

F)

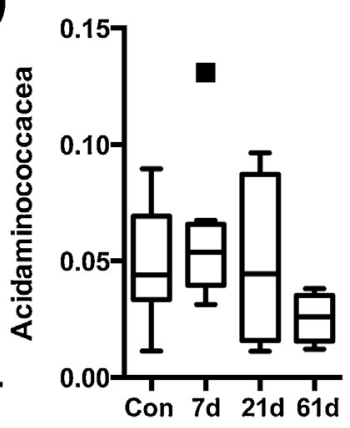

Fig. 3. Intestinal lymphatic obstruction changed the composition of the microbiome at family level.

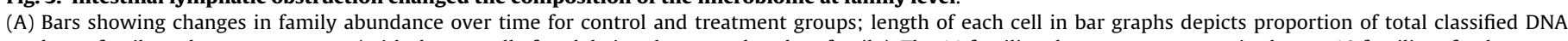

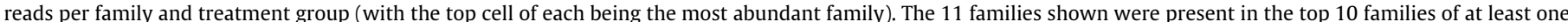

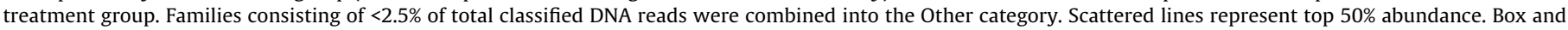

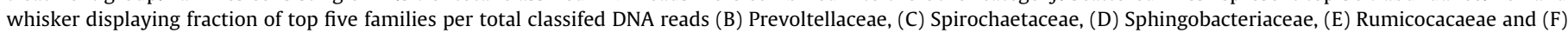

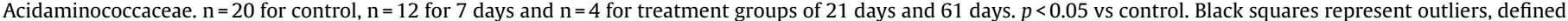
as values 1.5 times the interquartile range above the upper quartile.

Bars in Fig. 3B show changes in the family abundance over time for control and treatment groups. The length of each cell in this bar graph is proportionate to the total classified DNA reads per family within each treatment group. The 'box and whisker' plots (Fig. 3C-F) show the fraction of each of the top five families per total classified DNA reads in Prevotellaceae (3B), Spirochaetaceae (3C), Sphingobacteriaceae (3D), Rumicocacaeae (3E) and Acidaminococcaceae $(3 F)$. Prevotellaceae were significantly $\left({ }^{*} p<0.05\right)$ increased and Spirochaetaceae were significantly decreased $\left({ }^{*} p<0.05\right)$ at day 7 following ATLAS $\left({ }^{*} p<0.05\right)$ but not at other time points. 


\subsection{Intestinal lymphatic obstruction altered the composition of} the gut bacterial microbiome at the genus level

The abundance of thirteen bacterial genera changed dramatically as over time following ATLAS induction (Fig. 4). The 13 genera shown were present in the top 10 genera of at least one treatment group. Genera consisting of $<2.5 \%$ of total classified DNA reads were combined into the 'Other' category. Fig. 4A shows the changes in the abundance of each genera in control and over the 61 days postATLAS. As described above, the length of each cell in the bar graph in Fig. 4 shows proportion of total classified DNA reads per family and treatment group. From these 13 genera, the 'box and whisker' plots (Fig. 4B) show genera within the Bacteroides-PorphyromonasPrevotella (BPP) group for control and or treatment groups of 7 days, 21 days and 61 days post ATLAS induction, demonstrating an elevation in this grouping, which is consistent with development of dysbiosis being maximal at day 7 and is reversed by day 61 .

\subsection{Relationships between BPP species and each sample}

Finally, we used a 'Circos' plot (Fig. 5) to depict the proportion of those BPP species found within stool samples obtained from each of the subjects at different phases of the study, comparing control with 7, 21 and 61 days post-ATLAS. The experimental groups are shown on the top right third of the plot and each gradation in each experimental group represents an individual animal sample. The size of the link shown enlarges with the relative percentage of reads belonging to the sample found in that link. These results demonstrate a shift in the microbiome dysbiosis over the course of the 61 days post ATLAS vs. control.

\section{Discussion}

In our current study, we made several important observations regarding changes in the gut microbiome in the ATLAS model. We found that the gut microbiome 'contracted' in response to regional lymphatic obstruction in the first week, followed by a relative restoration of diversity at 21d and the microbial dysbiosis which occurs at 61 days.

We have previously reported that the induction of experimental colitis causes rapid and dramatic remodeling of intestinal lymphatics [20-22] and conversely, that disturbances in gut lymphatics (e.g. FOXC2 deletion (which produces a murine model of lymphedema distichiasis)) can hasten the onset and intensify colitic disease activity following induction of DSS colitis [23,24]. Clinically, Tonelli et al. [25], described lymphatic disturbances in CD, even recommending a surgical operation to restore normal clearance of intestinal lymph termed an 'epipoonoplasty'. However, whether or how such perturbations alter the intestinal microbiome, or whether a dysbiosis might be established by such manipulations that could support more persistent or chronic disease remains unclear. To the best of knowledge, no other study has evaluated whether experimentally-induced lymphostasis affects the composition of the gut microbiome and how this might contribute to intestinal stress seen in IBD or experimental colitis.

The distal GI tract contains large quantities of obligate anaerobic bacteria which increase by a factor of $10-100$ as one progresses from the ileum to the colon. In humans, the gut microbiome is represented by two main phyla, Firmicutes and Bacteroidetes which constitute the majority ( $\tilde{9} 0 \%)$ of the intestinal flora; these phyla are both obligate anaerobes. Apart from these conserved groupings of phyla, individual microbiomes show much more heterogeneity with up to 1000 different species found in a sample, but remarkably few species shared between individuals. The microbiome of the intestine is now recognized as a highly complex colony of commensal and occasionally pathogenic species whose composition can influence gut integrity [30], immunity [31] and metabolism [32]. In the setting of IBD, the recognition of the microbial influence on gut homeostasis has even led to the introduction of 'fecal transplants' as a means of adjusting the components of the gut flora to achieve therapeutic benefit. Several forms of hostcommensal/pathogen communication regulate intestinal epithelial barrier function [30] and may contribute to barrier disturbances which have been described in forms of IBD and other forms of intestinal inflammation. Therefore, several recent studies have revived investigations into the composition of the gut microbiome, its heterogeneity and influence on gut health and perturbations in disease.

The first finding in our study was a decrease in biodiversity in the AGM microbiome 7-dyas after induction of the ATLAS model. This timepoint also represents the peak of the inflammatory state [14]. In support of this, Ott et al. [33], reported that the diversity of the gut flora in CD was reduced to $50 \%$ of that seen in controls and was even further reduced (30\%) in UC. In particular, this loss of diversity reflected the loss of the anaerobic species Bacteroides, Eubacterium and Lactobacillus. Therefore, despite individual variations in microbial compositions, gut microbial 'crises 'appear to lead to an immediate 'bottleneck 'of fewer species. Interestingly, once an established pattern of microbial diversity is lost, particularly in adults, it is unclear how this balance can (if ever) be re-established. Therefore, if a specific microbial pattern governs local immunity and vascular ultrastructure, dysbiosis could lead to long-standing or lifelong immune dysregulation.

In addition, we found changes in four major microbial phyla (Bacterioidetes, Firmicutes, Spirochaetes, and Proteobacteria), with both Bacterioidetes and Proteobacteria being increased at 7 days. Bacterial toxins and biochemical changes in the intestinal lumen along with changes in gut motility may intensify injury, leading to a feed-forward 'greenhouse effect' which may generate a more extensively oxygenated gut environment which favors species which are facultatively anaerobes such as Bacterioidetes and Proteobacteria which have been described as a 'signature' of gut dysbiosis. Additionally, the significant decrease in the Firmicutes to Bacteroidetes ratio (which we found here) has been described as a key feature in both CD and dysbiosis [49]. At the family level, we found a significant increase in Prevotellaceae as well as a significant decrease in Spirochataceae. The genus Prevotella includes gram negative bacteria normally found in the oral and vaginal compartments. In the gut, the abundance of Prevotella can vary depending on diet such that protein-fat rich diets favor Bacteroides, whereas fiber and carbohydrate rich diets are associated with a greater proportion of Prevotella [28]. Normally, the prevailing obligate anaerobes e.g. Bacteroides and Prevotellaceae compete for dominance in the gut [29]. Here we show that dominant commensal taxa (such as Prevotellaceae and Bacteroides in the gut) often compete. When Bacteroides (or Ruminococcaceae) are suppressed, Prevotellaceae can expand to fill this niche, consistent with the significant elevation we observed in Prevotellaceae at day 7 in this model, but not at later time points.

Local inflammatory changes magnify blood perfusion of the intestinal mucosa, hence local increases in oxygen tension could profoundly affect the microbial environment to both suppress some commensals while eliminating others [34,35]. It is also known that the extreme hypoxia of the gut lumen favors the survival and proliferation of obligate anaerobic bacteria which dine upon complex carbohydrates not degraded within the upper gastrointestinal tract. In the large intestine, these complex carbohydrates are fermented into short-chain fatty acids (SCFA) e.g. acetic acid, propionic acid and butyric acid. These SCFA are largely utilized by gut epithelial cells which prefer SCFAs as carbon sources [36] providing links between epithelial barrier, oxygen levels and SCFA abundance. 

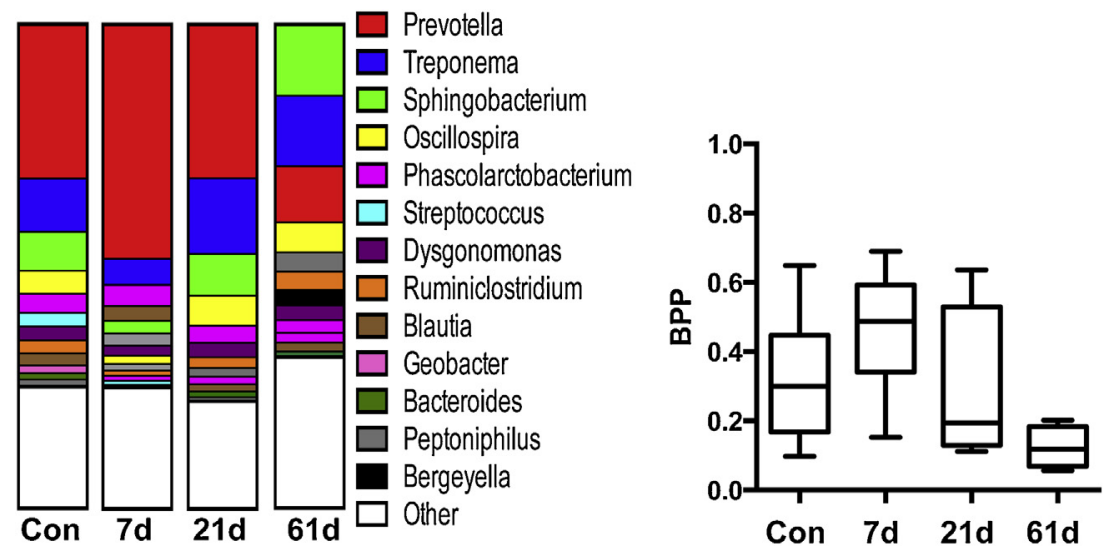

Fig. 4. Intestinal lymphatic obstruction changed the composition of the gut bacterial microbiome at the genus level.

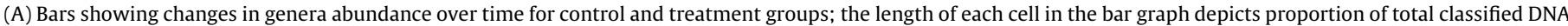

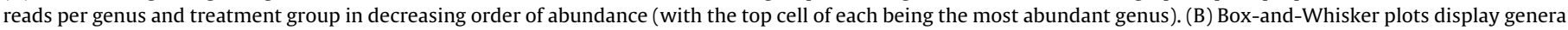
within the Bacteroides-Porphyromonas-Prevotella (BPP) group of $n=20$ for control, $n=12$ for 7 days and $n=4$ for treatment groups of 21 days and 61 days.

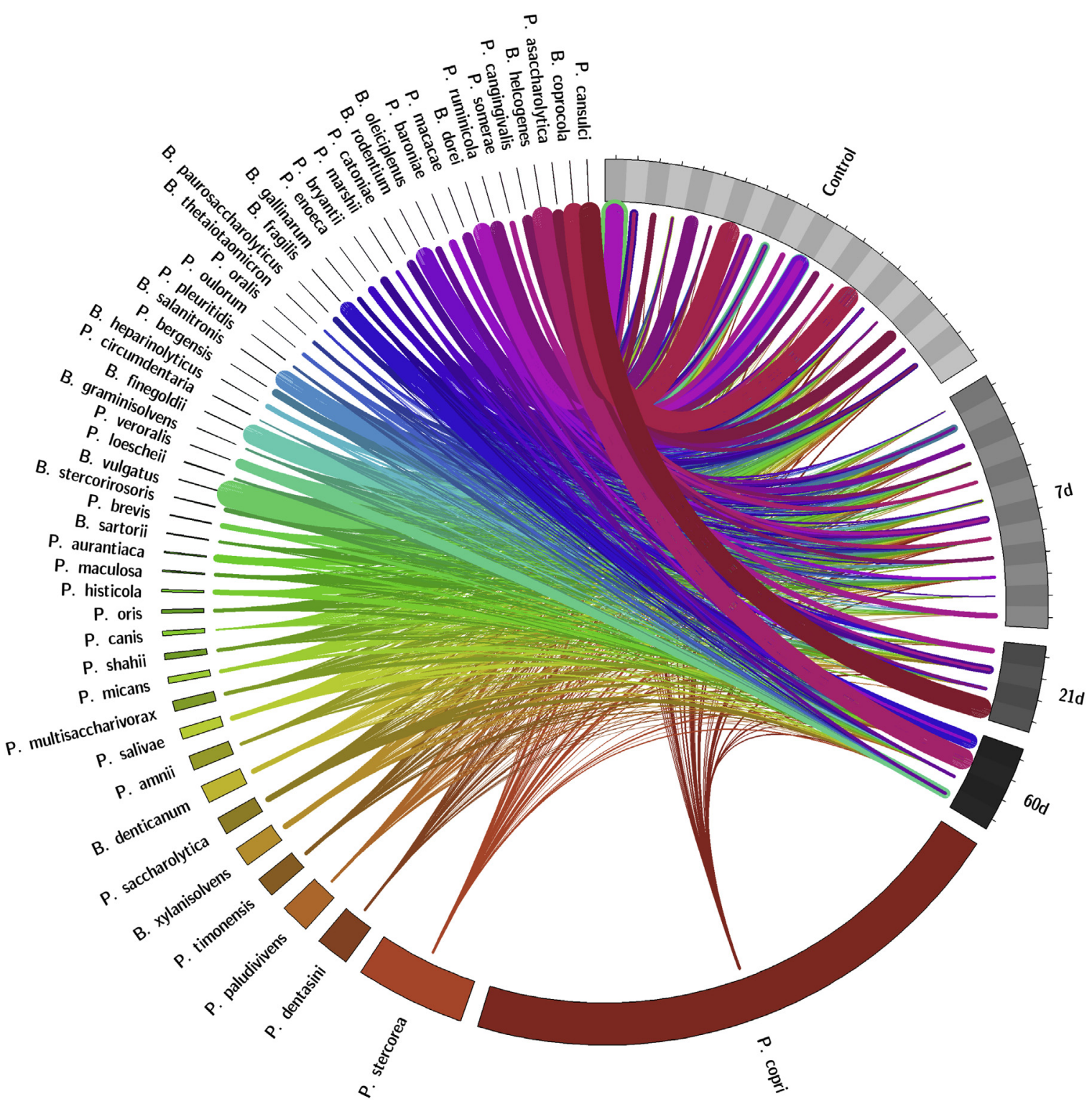

Fig. 5. Circos plot depicting the relative diversity and abundance of Bacteroides-Porphyromonas-Prevotella (BPP) species within each treatment group.

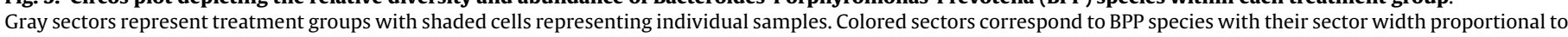

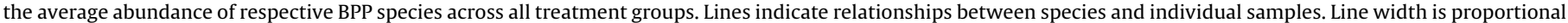
to the percentage of total DNA reads for that species belonging to the sample implicated in the link.

Please cite this article in press as: F. Becker, et al., Dynamic gut microbiome changes following regional intestinal lymphatic obstruction in primates, Pathophysiology (2019), https://doi.org/10.1016/j.pathophys.2019.06.004 
Importantly, as much as $10 \%$ of basal calories are provided by SCFAs. SCFAs, particularly butyrate, negatively regulate immune activation by suppressing immune cell derived Th1 cytokines (TNF $\alpha$, IFN $\gamma$, IL-6, IL-12) and nitric oxide and increasing Th2 cytokines e.g. IL-10 [37-40]. As SCFAs like butyric acid have been described as inhibitors of nuclear factor Kappa-B [41], there may be several potential links between dysbiosis, inflammatory activation and alterations in gut structure/function. Based on this concept, it has been suggested that dysbiosis (i.e. changes in the composition of the gut flora with a diminution of butyric acid generators) would lead to inflammatory changes which bring about inflammatory gut injury.

Although CD in humans is characterized by an increased microvessel density [45] (which closely correlates with disease severity), such vascular recruitment may reflect intermittent local hypoxia as a reaction to inflammation and oxygen consumption by immune cells. Hatoum et al. [46], have reported that gut mucosal perfusion is perturbed in IBD, with a concomittant hyperemia of the mesentery, bowel wall and serosa, but a relative hypoperfusion in the mucosa. Therefore, at least some phases of IBD may be hyperemic and relatively hyperoxic to gut commensals as a result of induced inflammation. Consequently, while thickening and hypervascularity in the intestinal wall correlate with IBD disease, perfusion may vary substantially and oxygen tension may vary substantially in IBD depending on disease state and gut region. Whether components of the microbiome are 'cleared 'by elevated oxygen tension is unknown. It has been shown that F. prausnitzii can be microaerobic, surviving low oxygen tensions provided they are provided with several co-factors e.g. thiols/flavins which they require to detoxify oxygen [47]. However, if perfusion is limited in IBD, these antioxidant factors could be depleted leading to a dropout of these factors and still provoke oxidant stress. Increased vascular perfusion may not be the only environmental source of oxygen affecting gut flora. Because intestinal lymphatics also participate in clearing oxygen laden water which passes across the epithelia into the interstitium [48] lymphatic transport failure may lead to an increase in stool water content (and hence oxygen) which could also increase oxygen permeation and microbiome intoxication.

Based on the structural and anatomic similarities to the human intestinal tract [15], we anticipated that lymphatic obstruction in the intestines of African green monkeys might produce a superior model which more accurately recapitulates the influence of lymphostasis on the changes in the microbiome than models that utilize rodents, lagomorphs and swine. Specifically, the intestinal anatomy of the African green monkey more closely matches the human intestinal components and segmental length, as well as the blood and lymphatic supplies. Perhaps most importantly the derivation of nutrition and the reliance of non-human species (rats, mice, rabbits) on fermentation may mean that rodents and lagomorphs are not good models in which to compare intestinal stress. Although all are monogastric omnivores, rats and mice are well-adapted to carry out fermentation $[5,19]$. How such differences might influence the tolerability of short-term alterations in the gut microbiome and how these differences contribute to the pathophysiology of inflammatory diseases is completely unknown, but support the greater relevance of non-human primates for such studies.

\section{Conclusions}

Using the 'ATLAS' model of CD to study how the gut microbiome changes following regional surgically-induced obstruction of gut lymphatics, significant differences were found between the control versus day 7 and day 61 groups with respect to total classified reads, reads associated with Prevotellaceae and reads associated with the BPP group. These findings are the first report linking lymphatic structural/function changes with alterations in the gut microbiome as they may relate to the pathophysiology of $C D$.

\section{Acknowledgements}

Research reported in this publication were supported by the Feist-Weiler Cancer Center (FWCC) Eastern-Star Award (JSA) and a supplement award from the FWCC (JSA), as well as from the Department of Defense PR100451 'Lymphatic Vascular-Based Therapy for IBD' (JSA) and a COBRE award (RS) National Institute of General Medical Sciences of the National Institutes of Health under Award Number P30GM110703.

\section{References}

[1] F. Becker, P. Yi, M. Al-Kofahi, V.C. Ganta, J. Morris, J.S. Alexander, Lymphatic dysregulation in intestinal inflammation: new insights into inflammatory bowel disease pathomechanisms, Lymphology 47 (2014) 3-27.

[2] D.J. Chivers, C.M. Hladik, Morphology of the gastrointestinal tract in primates: comparisons with other mammals in relation to diet, J. Morphol. 166 (1980) 337-386, http://dx.doi.org/10.1002/jmor.1051661306.

[3] M. Wos-Oxley, A. Bleich, A.P. Oxley, S. Kahl, L.M. Janus, A. Smoczek, H. Nahrstedt, M.C. Pils, S. Taudien, M. Platzer, H.J. Hedrich, E. Medina, D.H Pieper, Comparative evaluation of establishing a human gut microbial community within rodent models, Gut Microbes 3 (2012) 234-249, http://dx doi.org/10.4161/gmic.19934.

[4] T.L. Nguyen, S. Vieira-Silva, A. Liston, J. Raes, How informative is the mouse for human gut microbiota research? Dis. Model. Mech. 8 (2015) 1-16, http://dx. doi.org/10.1242/dmm.017400.

[5] A.H. Moeller, Y. Li, E. Mpoudi Ngole, S. Ahuka-Mundeke, E.V. Lonsdorf, A.E. Pusey, M. Peeters, B.H. Hahn, H. Ochman, Rapid changes in the gut microbiome during human evolution, Proc. Natl. Acad. Sci. U. S. A. 111 (2014) 16431-16435, http://dx.doi.org/10.1073/pnas.1419136111.

[6] D.C. Rapozo, C. Bernardazzi, H.S. de Souza, Diet and microbiota in inflammatory bowel disease: the gut in disharmony, World J. Gastroenterol. 23 (2017) 2124-2140, http://dx.doi.org/10.3748/wjg.v23.i12.2124.

[7] K. Matsouka, T. Kanai, The gut microbiota and inflammatory bowel disease, Semin. Immunopathol. 37 (2015) 47-55, http://dx.doi.org/10.1007/s00281014-0454-4.

[8] M. Patel, J. Olinde, A. Tatum, C.V. Ganta, W.E. Cromer, A.R. Sheth, M.H. Jennings, J.M. Mathis, T. Testerman, P.A. Jordan, K. Manas, C.P. Monceaux, J.S. Alexander, Gut sterilization in experimental colitis leukocyte mediated colon injury, and effects on angiogenesis/lymphangiogenesis, Open J. Gastroenterol. 3 (2013) 12-24, http://dx.doi.org/10.4236/ojgas.2013.31003.

[9] P.M. Munyaka, M.F. Rabbi, E. Khafipour, J.E. Ghia, Acute dextran sulfate sodium (DSS)-induced colitis promotes gut microbial dysbiosis in mice, J. Basic Microbiol. 56 (2016) 986-998, http://dx.doi.org/10.1002/jobm.201500726.

[10] K.A. Kigerl, J.C. Hall, L. Wang, X. Mo, Z. Yu, P.G. Popovich, Gut dysbiosis impairs recovery after spinal cord injury, J. Exp. Med. 213 (2016) 2613-2620, http:// dx.doi.org/10.1084/jem.20151345.

[11] J. Hu, H. Luo, J. Wang, W. Tang, J. Lu, S. Wu, Z. Xiong, G. Yang, Z. Chen, T. Lan, H Zhou, J. Nie, Y. Jiang, P. Chen, Enteric dysbiosis-linked gut barrier disruption triggers early renal injury induced by chronic high salt feeding in mice, Exp. Mol. Med. 49 (2017) e370, http://dx.doi.org/10.1038/emm.2017.122.

[12] J.M. Mariadason, D.H. Barkla, P.R. Gibson, Effect of short-chain fatty acids on paracellular permeability in Caco-2 intestinal epithelium model, Am. J. Physiol. 272 (1997) G705-712, http://dx.doi.org/10.1152/ajpgi.1997.272.4. G705.

[13] G. Goverse, R. Molenaar, L. Macia, J. Tan, M.N. Erkelens, T. Konijn, M. Knippenberg, E.C. Cook, D. Hanekamp, M. Veldhoen, A. Hartog, G. Roeselers, C.R. Mackay, R.E. Mebius, Diet-derived short chain fatty acids stimulate intestinal epithelial cells to induce mucosal tolerogenic dendritic cells, J. Immunol. 198 (2017) 2172-2181, http://dx.doi.org/10.4049/jimmunol. 1610165.

[14] F. Becker, E. Romero, J.D.L. Hasselschwert, B. Dray, J. Vanchiere, J. Fontenot, W. Yun, M. Musso, P.C. Norris, C.N. Serhan, J.S. Alexander, F.N.E. Gavins, Endogenous specialized pro-resolving mediator profiles in a novel experimental model of lymphatic obstruction and intestinal inflammation in African Green Monkeys,. Am J. Path. (2019).

[15] T.V. Kalima, Experimental lymphatic obstruction in the ileum, Ann. Chir. Gynaecol. Fenn. 59 (1970) 187-201.

[16] Illumina, Instructions for Using the 16S Metagenomics App. (15055861 A), 2014.

[17] Q. Wang, G.M. Garrity, J.M. Tiedje, J.R. Cole, Naïve Bayesian classifier for rapid assignment of rRNA sequences into the new bacterial taxonomy, Appl. Environ. Microbiol. 16 (2007) 5261-5267, http://dx.doi.org/10.1128/AEM. 00062-07.

[18] A. Klindworth, E. Pruesse, T. Schweer, J. Peplles, C. Quast, M. Horn, F.O. Glöckner, Evaluation of general 16S ribosomal RNA gene PCR primers for 
classical and next-generation sequencing-based diversity studies, Nucleic Acids Res. 41 (2013) e1, http://dx.doi.org/10.1093/nar/gks808.

[19] M. Nyman, N.G. Asp, Fermentation of dietary fibre components in the rat intestinal tract, Br. J. Nutr. 47 (1982) 357-366.

[20] J.S. Alexander, G.V. Chaitanya, M.B. Grisham, M. Boktor, Emerging roles of lymphatics in inflammatory bowel disease, Ann. N. Y. Acad. Sci. 1207 (2010) E75-E85, http://dx.doi.org/10.1111/j.1749- 6632.2010.05757.

[21] J.S. Alexander, V.C. Ganta, P.A. Jordan, M.H. Witte, Gastrointestinal lymphatics in health and disease, Pathophysiology 17 (2010) 315-335, http://dx.doi.org/ 10.1016/j.pathophys.2009.09.003.

[22] W.E. Cromer, C.V. Ganta, T.M. Patel, J. Traylor, C.G. Kevil, J.S. Alexander, J.M. Mathis, VEGF-A isoform modulation in a preclinical TNBS model of ulcerative colitis: protective effects of a VEGF164b therapy, J. Transl. Med. 11 (2013) 207, http://dx.doi.org/10.1186/1479-5876-11-207.

[23] V.C. Ganta, W. Cromer, G.L. Mills, J. Traylor, M. Jennings, S. Daley, B. Clark, J.M. Mathis, M. Bernas, M. Boktor, P. Jordan, M. Witte, J.S. Alexander, Angiopoietin-2 in experimental colitis, Inflamm. Bowel Dis. 16 (2010) 1029-1039, http://dx.doi.org/10.1002/ibd.21150.

[24] F. Becker, S. Potepalov, R. Shehzahdi, M. Bernas, M. Witte, F. Abreo, J. Traylor, W.A. Orr, I. Tsunoda, J.S. Alexander, Downregulation of FoxC2 increased susceptibility to experimental colitis: influence of lymphatic drainage function? Inflamm. Bowel Dis. 21 (2015) 1282-1296, http://dx.doi.org/10. 1097/MIB.0000000000000371.

[25] F. Tonelli, F. Giudici, G. Liscia, Is lymphatic status related to regression of inflammation in Crohn's disease? World J Gastrointest Surg. 4 (2012) 228-233, http://dx.doi.org/10.4240/wjgs.v4.i10.228.

[28] G.D. Wu, J. Chen, C. Hoffmann, K. Bittinger, Y.Y. Chen, S.A. Keilbaugh, M. Bewtra, D. Knights, W.A. Walters, R. Knight, R. Sinha, E. Gilroy, K. Gupta, R. Baldassano, L. Nessel, H. Li, F.D. Bushman, J.D. Lewis, Linking long-term dietary patterns with gut microbial enterotypes, Science 334 (2011) 105-108, http://dx.doi.org/10.1126/science.1208344.

[29] K. Faust, J.F. Sathirapongsasuti, J. Izard, N. Segata, D. Gevers, J. Raes, C. Huttenhower, Microbial Co-occurrence relationships in the human microbiome, PLoS Comput. Biol. 8 (2012) e1002616, http://dx.doi.org/10. 1371/journal.pcbi.1002616.

[30] G.C. Hansson, Role of mucus layers in gut infection and inflammation, Curr. Opin. Microbiol. 15 (2012) 57-62, http://dx.doi.org/10.1016/j.mib.2011.11. 002.

[31] A. Hayashi, T. Sato, N. Kamada, Y. Mikami, K. Matsuoka, T. Hisamatsu, T. Hibi, A. Roers, H. Yagita, T. Ohteki, A. Yoshimura, T. Kanai, A single strain of Clostridium butyricum induces intestinal IL-10-producing macrophages to suppress acute experimental colitis in mice, Cell Host Microbe 13 (2013) 711-722, http://dx.doi.org/10.1016/j.chom.2013.05.013.

[32] J. Parekh, L.A. Balart, D.A. Johnson, The influence of the gut microbiome on obesity, metabolic syndrome and gastrointestinal disease, Clin. Transl. Gastroenterol. 6 (2015) e91, http://dx.doi.org/10.1038/ctg.2015.16.

[33] S.J. Ott, M. Musfeldt, D.F. Wenderoth, J. Hampe, O. Brant, U.R. Folsch, K.N. Timmis, S. Schreiber, Reduction in diversity of the colonic mucosa associated bacterial microflora in patients with active inflammatory bowel disease, Gut 53 (2004) 685-693.

[34] P.R. Kvietys, T. Miller, D.N. Granger, Intrinsic control of colonic blood flow and oxygenation, Am. J. Physiol. 238 (1980) G478-484, http://dx.doi.org/10.1152/ ajpgi.1980.238.6.G478.
[35] L. Rigottier-Gois, Dysbiosis in inflammatory bowel diseases: the oxygen hypothesis, ISME J. 7 (2013) 1256-1261, http://dx.doi.org/10.1038/ismej. 2013.80.

[36] M.A. Vinolo, G.J. Ferguson, S. Kulkarni, G. Damoulakis, K. Anderson, M. Bohlooly-Y, L. Stephens, P.T. Hawkins, R. Curi, SCFAs induce mouse neutrophil chemotaxis through the GPR43 receptor, PLoS One 6 (2011) e21205, http:// dx.doi.org/10.1371/journal.pone.0021205.

[37] J.S. Park, E.J. Lee, J.C. Lee, W.K. Kim, H.S. Kim, Anti-inflammatory effects of short chain fatty acids in IFN-gamma-stimulated RAW 264.7 murine macrophage cells: involvement of NF-kappaB and ERK signaling pathways, Int. Immunopharmacol. 7 (2007) 70-77, http://dx.doi.org/10.1371/10.1016/j. intimp.2006.08.015.

[38] D. Chakravortty, N. Koide, Y. Kato, T. Sugiyama, M.M. Mu, T. Yoshida, T. Yokochi, The inhibitory action of butyrate on lipopolysaccharide-induced nitric oxide production in RAW 264.7 murine macrophage cells, J. Endotoxin Res. 6 (2000) 243-247.

[39] M. Usami, K. Kishimoto, A. Ohata, M. Miyoshi, M. Aoyama, Y. Fueda, J. Kotani, Butyrate and trichostatin A attenuate nuclear factor kappaB activation and tumor necrosis factor alpha secretion and increase prostaglandin E2 secretion in human peripheral blood mononuclear cells, Nutr. Res. 28 (2008) 321-328, http://dx.doi.org/10.1016/j.nutres.2008.02.012.

[40] D. Saemann, G.A. Böhmig, C.H. Osterreicher, H. Burtscher, O. Parolini, C. Diakos, J. Stöckl, W.H. Hörl, G.J. Zlabinger, Anti-inflammatory effects of sodium butyrate on human monocytes: potent inhibition of IL-12 and up-regulation of IL-10 production, FASEB J. 14 (2000) 2380-2382, http://dx. doi.org/10.1096/fj.00-0359fje.

[41] J.P. Segain, D. Raingeard de la Blétière, A. Bourreille, V. Leray, N. Gervois, C. Rosales, L. Ferrier, C. Bonnet, H.M. Blottière, J.P. Galmiche, Butyrate inhibits inflammatory responses through NFkappaB inhibition: implications for Crohn's disease, Gut 47 (2000) 397-403.

[45] J. Spalinger, H. Patriquin, M.C. Miron, G. Marx, D. Herzog, J. Dubois, M. Dubinsky, E.G. Seidman, Doppler US in patients with crohn disease: vessel density in the diseased bowel reflects disease activity, Radiology 217 (2000) 787-791, http://dx.doi.org/10.1148/radiology.217.3.r00dc19787.

[46] O.A. Hatoum, D.G. Binion, D.D. Gutterman, Paradox of simultaneous intestinal ischaemia and hyperaemia in inflammatory bowel disease, Eur. J. Clin. Invest. 35 (2005) 599-619, http://dx.doi.org/10.1111/j.1365-2362.2005.01567.x.

[47] M.T. Khan, S.H. Duncan, A.J. Stams, J.M. van Dijl, H.J. Flint, H.J. Harmsen, The gut anaerobe Faecalibacterium prausnitzii uses an extracellular electron shuttle to grow at oxic-anoxic interphases, ISME J. 6 (2012) 1578-1585, http://dx.doi.org/10.1038/ismej.2012.5.

[48] K.D. Crissinger, P.R. Kvietys, D.N. Granger, Pathophysiology of gastrointestinal mucosal permeability, J. Intern. Med. Suppl. 732 (1990) 145-154.

[49] J. Kabeerdoss, P. Jayakanthan, S. Pugazhendhi, B.S. Ramakrishna, Alterations of mucosal microbiota in the colon of patients with inflammatory bowel disease revealed by real time polymerase chain reaction amplification of $16 \mathrm{~S}$ ribosomal ribonucleic acid, Indian J. Med. Res. 142 (1) (2015) 23-32, http://dx. doi.org/10.4103/0971-5916.162091, PubMed PMID: 26261163; PubMed Central PMCID: PMC4557246. 